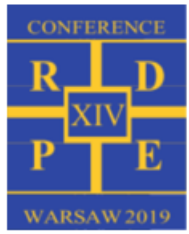

\title{
Thorium application in the medium-sized sodium-cooled fast reactor
}

\author{
Eeshu Raaj Saasthaa A rumuga K umar ${ }^{1}$, Piotr Darnowski ${ }^{1 *}$, Mihir K iritbhai Pancholi ${ }^{1}$ and Aleksandra Dzido $^{1}$ \\ ${ }^{1}$ W arsaw University of Technology, Institute of Heat Engineering, N owowiejska 21/25,00-665, W arsaw, Poland \\ ${ }^{*}$ Corresponding author: piotr.darnowski@pw.edu.pl
}

\begin{abstract}
The report presents an analysis of the medium-sized Sodium-Cooled Fast Reactor (SFR) core with Thorium-based Mixed-Oxide fuel. The introduction of Transuranics (TRU) to the fuel was to allow long-lived nuclear waste incineration. The studied core is based on the modified Advanced Burner Reactor (ABR) 1000M W th core design, which was analysed in the OECD/NEA "Benchmark for Neutronic Analysis of Sodium-Cooled Fast Reactor Cores with Various Fuel Types and Core Sizes". The full-core simulations with SERPENT 2.1.31 Monte Carlo computer code and ENDF library were performed, including static criticality and fuel burnup calculations for five fuel cycles. The core inventories at the Beginning of Cycle (BOC) and End of Cycle (EOC) were studied, and the impact of thorium fuel was assessed. The proposed core design is a burner reactor which uses thorium fuel. The excess core reactivity stays positive for long time despite large net consumption of transuranic elements as new fissile U ranium 233 is constantly breed from Thorium 232. B reeding of uranium allows longer fuel cycles.
\end{abstract}

\section{Introduction}

In 2002, the Generation IV International Forum (GIF) was founded to propel the future of the Nuclear Power industry through international co-operation using knowledge exchange and research. The activities of which are continued till date, the primary goal of the GIF was to design the next generation of reactors that will enable us to achieve the sustainable and efficient usage of uranium resources. The GIF identified six potential reactor designs that meet its basic goal which will also provide the highest level of nuclear safety, resistance to nuclear proliferation, minimization of radioactive waste production and other applications such as industrial heat, hydrogen production and so on [1],[2].

One of the most promising and matured Gen-IV design is the Sodium Cooled Fast Reactor (SFR). M any countries, such as R ussia, France, China and India, have shown their interest in SFR technology building more than half a century of experience. As of 2019, there are six reactors under operation: the Russian (BN -600, BN800, BOR-60), the Chinese (CEFR) and the Indian (PFBR, FBTR) SFRs [2]. The most advanced operating Generation-IV reactor is the Russian BN-800 which started its operation in 2016. The European project ASTRID based in France was unfortunately frozen in later stage of its development in 2019 [3]. The USA recently raised interest in the SFR technology, as in March 2019 the Department of Energy (DOE) announced development of sodium $V$ ersatile Test Reactor (VTR) based on the GE-Hitachi PRISM technology [4].

Thorium was identified as a prospective nuclear fuel and an alternative to uranium way back in the 1950s. Thorium naturally occurs as Th-232 and is about three times more abundant than uranium [5]. Currently, there is no commercial-scale thorium fuelled nuclear reactors under operation, meaning all that resource is left practically unused for energy production. One of the main reasons is that thorium has no fissile isotope; therefore it needs either uranium or plutonium as drivers for reactor cores to initiate breeding of fissile U233. It can be breed in both fast and thermal spectrum which makes it different than uranium-plutonium which demands fast neutron spectrum for breeding [2]. In the early days of nuclear power, the development was directed into usage of uranium. Partly because early reactors were built to produce military plutonium and thorium produces weapons-grade material, which is difficult to handle, which means it is a profit when you look from the proliferation resistance point of view. Thus, uranium technology was matured and preferred to be used as it was more problematic to start development of new thorium technology with the resources back then. Renewal interest in new reactor designs and trend of decarbonisation makes the time more apt for thorium than ever before.

Thorium's ability to breed fissile material and produce relatively small traces of plutonium and minor actinides makes it considerable fuel for the Gen-IV requirements creating a larger interest to use thoriumbased nuclear fuel [2],[5]. Most of the reactor designs suit thorium as a fuel, especially Molten Salt Reactors and High-Temperature Reactors, but it can also be utilized in Sodium Cooled Fast Reactors. A lthough, it is believed that the MSR will be most efficient, but the technology is still in early development phase, and it will take many years of research before it can make it to the commercial stage [6]. 
There are arguments that the application of thorium in fast reactors is not very competitive when comparing its application in thermal designs. Those arguments are mainly due to the possibility to use the large depleted uranium stockpiles, which are the heritage of the Cold $W$ ar and by-product of enrichment process. In the future, in the event of uranium shortage thorium can be a serious alternative and its application in fast reactors should not be discarded. In their paper [6], Gyorgy and Czifrus studied different Gen-IV designs where they showed that fast spectrum reactors (SFR and LFR) have neutronic advantages in comparison to other designs using thorium. Moreover, due to its lower MA production thorium can be used in nuclear waste burner reactors which leads to the scope and motivation of this paper.

The inspiration of the current work was the paper by Gyorgy and Czifrus [7], who studied the application of thorium in large 3600M W th core based on OECD design with oxide fuel which was proposed in "Benchmark for Neutronic Analysis of Sodium-Cooled Fast Reactor Cores with Various Fuel Types and Core Sizes" [8]. On the contrary, in this paper, the OECD $1000 \mathrm{M}$ W th medium-size oxide core design was used as a reference. This design is based on the Advanced Burner Reactor (ABR) [9],[10], a core dedicated for nuclear waste incineration and electricity production studied for several years by Argonne National Laboratory [9].

The main objective of this project was to propose a core design that can use mixed oxide fuel with thorium, plutonium and minor actinides [11] and that can be used for long-lived nuclear waste incineration. Then, the objective was to study the developed core from the neutronic point of view. In this paper an emphasis was put on the isotopic inventory changes in time.

\section{Methodology}

\subsection{Tools}

The three-dimensional continuous energy Monte Carlo reactor physics code, the Serpent 2 was applied [12][14]. It solves neutron-transport equation allowing to study static-criticality properties of a core, and it also allows to simulate fuel depletion using the solution of $B$ ateman equations. All calculations were performed using the ENDF-VII nuclear data library.

\subsection{Studied reactor cores}

The reference core was the 1000M W th oxide core with (U,TRU)02 fuel described in the SFR OECD $B$ enchmark [15]. It was three-zone design with 30 inner, 90 middle and 60 outer fuel assemblies. The reference core was solved using the Serpent 2.1.28 code in a previous study [16], which served as the base for our current expedition. The thorium core studied in this work has the same number of assemblies and dimensions but the core pattern, fuel materials and enrichment zones are different - see Figure 1. The design of assemblies for reflectors, control and structure are the same as the previous study [16].

The radial core layout consisted of two rings: inner and outer core rings with slightly higher fuel enrichment in the outer core to distinguish them. The number of inner and outer assemblies were 108 and 72 respectively. In total 180 fuel assemblies were equipped in the core design. Such a design was chosen firstly, to have enough inner irradiated fuel for the next cycle. Secondly, the inner core should contain more thorium Th232 due to the fact that neutron population is higher towards the centre of the core leading to higher transmutation to Pa233 which indeed would lead to higher production of fissile U 233 [7].

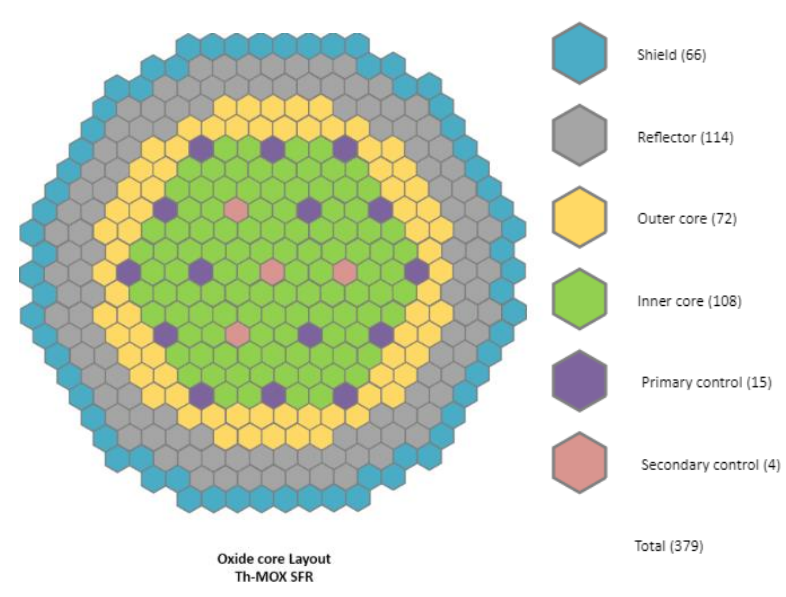

Fig. 1. 1000M W th Th-M OX SFR core map.

\subsection{Thorium fuel}

The fuel type applied was Thorium M ixed Oxide fuel. It contains reprocessed spent nuclear fuel that contains Plutonium but also Minor Actinides mixed with thorium feed. It can be abbreviated as $(\mathrm{Th}, \mathrm{TRU}) \mathrm{O}_{2}$ or $\left(\mathrm{Th}, \mathrm{Pu}+\mathrm{MA} \mathrm{O}_{2}\right.$. The TRU fuel isotopic vector is the same as oxide charge fuel used by $\mathrm{Kim}$ et al. in their ABR study [9] (see Table 2). Kim's mixture was used in OECD study as a fresh fuel in fuel cycle calculations to prepare the equilibrium core used in the benchmark [8].

Studies have shown that thorium mixed fuel can be used in nuclear reactors effectively, and in this work, we extrapolate this result to TRU based mixed oxide [6],[11]. Thorium charge composition is based on the natural thorium mixture.

The fuel pin design and dimensions are the same as in the reference core. It was designed with a smeared density of $85 \%$, to allow the possibility of fuel swelling during normal operation. For simplicity, Oxide-to-M etal ratio was assumed to be 2.0 . The thorium fuel density was estimated using the thorium thermophysical correlations implemented in the X-Core $M$ atlab Toolbox [17]. Thorium in the inner core had an atomic fraction of $73 \%$ and $72 \%$ in the outer core. The remaining $(27 \%$ and $28 \%$ ) consisted transuranic elements (TRU), both plutonium and minor actinides. It is to be noted that Pu239 and Pu241 are the main fissile isotopes in the vector shown in Table 2 . These two are the driver fuel for the core, what is more, is that the fissile isotopes 
fraction is less than $50 \%$ of the total TRU fraction. Which means the enrichment for the fuel chosen is less than $20 \%$ thus abiding the non-proliferation standards.

Table 2. Isotope vectors used in Th-MOX/TRU fuel charge and at BOL. TRU fuel vector is based on [9].

\begin{tabular}{|c|c|}
\hline Isotope & Atomic Fraction [-] \\
\hline \multicolumn{2}{|r|}{ Thorium } \\
\hline Th230 & 0.0002 \\
\hline Th232 & 0.9998 \\
\hline \multicolumn{2}{|r|}{ Oxygen } \\
\hline O16 & 0.99757 \\
\hline $\mathrm{O} 17$ & 0.00243 \\
\hline \multicolumn{2}{|r|}{ TRU } \\
\hline Np237 & 0.015 \\
\hline $\mathrm{Pu} 238$ & 0.031 \\
\hline $\mathrm{Pu} 239$ & 0.411 \\
\hline $\mathrm{Pu} 240$ & 0.313 \\
\hline Pu241 & 0.057 \\
\hline $\mathrm{Pu} 242$ & 0.081 \\
\hline Am241 & 0.036 \\
\hline $\mathrm{Am} 242 \mathrm{~m}$ & 0.002 \\
\hline Am243 & 0.026 \\
\hline $\mathrm{Cm} 243$ & 0.0 \\
\hline $\mathrm{Cm} 244$ & 0.018 \\
\hline $\mathrm{Cm} 245$ & 0.005 \\
\hline $\mathrm{Cm} 246$ & 0.003 \\
\hline
\end{tabular}

Enrichment was selected based on initial criticality and fuel burnup studies. The goal was to obtain BOL core eigenvalue comparable to the multiplication factor (k-eff) of the reference core and to keep the reactivity margin to critical state being at least of 0.001 SD guaranteeing that the reactor is operational, and the effect of Monte Carlo statistical error impact is minimized.

\subsection{Fuel cycle simulations}

The core was assumed to run a cycle with 328.5 effective full-power days, corresponding to one year with $90 \%$ capacity factor. Eight burnup steps were applied corresponding to: $0,1,5,25,75,115,185,250$ and 328.5 full power days with a power density 0.06521 $\mathrm{kW} / \mathrm{gHM}$ throughout the cycle. After each cycle a 90 day decay period was assumed to let the decay of protactinium into uranium and perform proper core reloading (Figure 2). In total five cycles were computed to estimate reaching the equilibrium core state. Static criticality calculations were performed to check whether the excess reactivity stays large enough to allow operation.

The fresh fuel was introduced in the first cycle, and after the end of cycle (EOC) inner fuel was reshuffled to the outer core upon completion of the decay period (see Figure 2). The same reshuffling pattern was used throughout the remaining cycles. In other words, the fuel resides two cycles in the core, one cycle in the inner core; then it is moved to the outer core and finally to reprocessing or spent fuel storage. The one cycle irradiated fuel composition was decayed using the decay function of SERPENT. The decay steps chosen were $1,5,15,25,35,45,65,75$ and 90 days. The fission products and the HM isotopes tracked during the decay process are essential for calculating the irradiated fuel composition which will be the fuel input for the outer assemblies in the following cycle. The decay process entirely focuses on the decay of Pa233 to U 233; the FP tracked were same as that from our previous study [16].

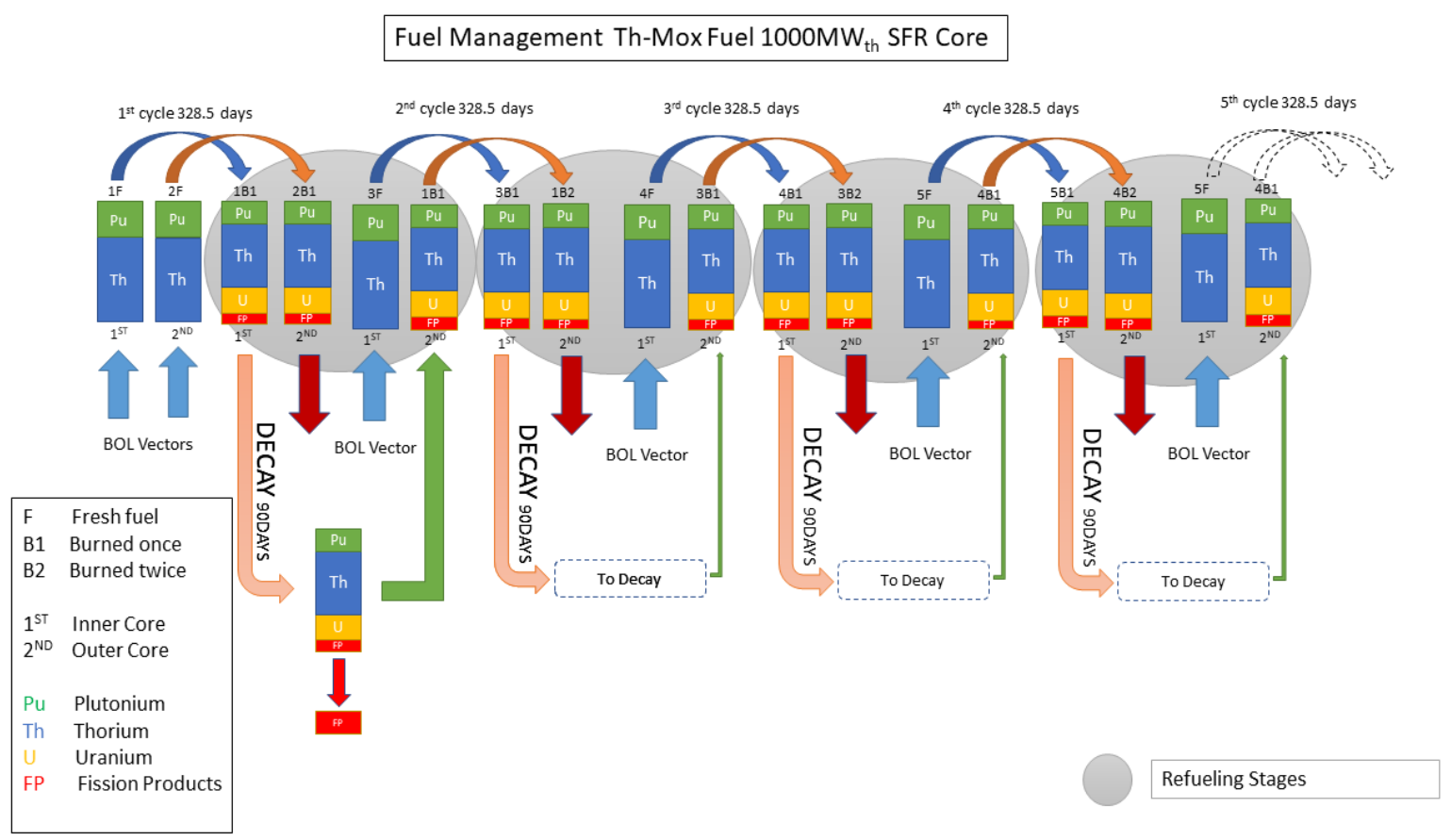

Fig. 2. Fuel management overview. Flowchart inspired from [18]. 
Figure 2 shows the fuel management strategy implemented in this work and such a strategy was chosen comes down to two primary objectives: the possibility to use the plutonium (and minor actinides) stockpiles appropriately and to reduce the usage of fresh fuel thereby reducing fuel production effort. Bearing in mind the scope of the paper, the applied approach was simplified to study only the fuel usage in the reactor, not the whole fuel cycle with spent fuel and reprocessing strategy. In future research it is possible to address various applicable cycles in more detail. M oreover, the studied core can operate much longer without reloading adding flexibility to the fuel management cycle, and fuel can be reloaded more than once as achieved burnup is relatively low.

For fuel burnup calculations, core was divided into two radial zones and five axial zones, hence, totally ten different burnup zones. Depletion computations for five fuel cycles were performed with relatively low neutron population, as this work is a preliminary study. Therefore, a neutron population of 30000 in 250 cycles and 100 inactive cycles was introduced to establish a balance between the computational time and power at hand while making sure that the maximum Relative Statistical Error (RSE) did not exceed 0.00057 .

\section{RESULTS AND DISCUSSION}

The effective multiplication factor (k-eff or eigenvalue) along with the change in the fuel inventory were observed closely. The time evolution for five cycles is shown in Figure 3, and core inventory at BOC and $\mathrm{EOC}$ is presented in Table 1.

The core is approaching the equilibrium state during the 2nd cycle; hence, both k-eff and core inventory above second cycle are similar. Results are compared with reference equilibrium core - light blue in Figure 3 [16]. The BOC eigenvalue is close to the reference core, as it was one of the design constraints during selection of the core configuration. One can observe that the k-eff for EOC state is also very close to the reference core as burnup configuration was similar.

The substantial initial drop in eigenvalue for each cycle (Figure 3 ) is due to the production of Pa233, neutron poison from Th232. Pa233 decays to U 233, until it reaches saturation concentration after which the poisoning effect stays constant. In every cycle, Pa233 reaches an equilibrium after $\sim 100$ days and then breeding of fissile U 233 continues steadily introducing additional reactivity to the core.

The conversion ratio (CR) for each cycle was $\sim 0.96$ (Table 1). This parameter is the ratio of fissile materials breeding rate per fissile materials consumption rate, and it met the criteria for sustainable design $(C R \sim 1)$. Generally, for a core to be a breeder the CR should be higher than one, for break-even (ideal converter) core it should be close to one and for converter core $C R$ is lower than one.

The reference $A B R$ core conversion ratio was slightly lower $C R \sim 0.9$ and $C R_{\text {TRU }} \sim 0.75$. The behaviour of the reference core is different as eigenvalue is steadily decreasing during the cycle (Figure 3 ) as fissile materials are consumed. For the studied thorium core after the decay period, the eigenvalue stays constant, and it is caused by the breeding of fissile U233 which compensates burnup.

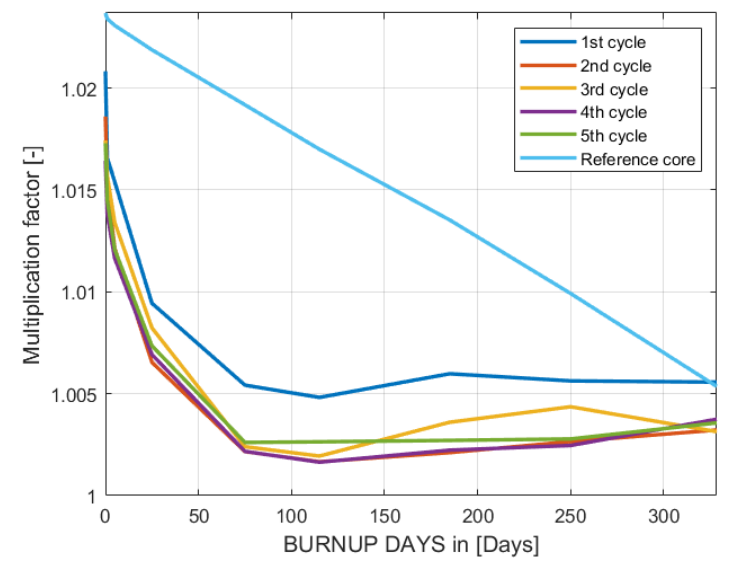

Fig. 3. Effective multiplication factor for five cycles of thorium core compared with reference uranium-plutonium core.

The overall mass balance for basic elements and groups of elements, between the $\mathrm{BOC}$ and $\mathrm{EOC}$ (at shutdown) is shown in Table 1 . We can observe that

Table 1. Cumulative masses of isotope groups in [kg]. TRU consumption is in [kg/TWh]. CR is dimensionless [-].

\begin{tabular}{ccccccccccc}
\hline Cycle & \multicolumn{2}{c}{$\mathbf{1}$} & \multicolumn{2}{c}{$\mathbf{2}$} & \multicolumn{2}{c}{$\mathbf{3}$} & \multicolumn{2}{c}{$\mathbf{4}$} & \multicolumn{2}{c}{$\mathbf{5}$} \\
\hline Stage & BOC & EOC & BOC & EOC & BOC & EOC & BOC & EOC & BOC & EOC \\
\hline U233+Pu239+Pu241 & 1939 & 1876 & 1906 & 1848 & 1902 & 1843 & 1906 & 1865 & 1901 & 1841 \\
Fissile & 2507 & 2416 & 2456 & 2375 & 2387 & 2367 & 2456 & 2397 & 2387 & 2366 \\
U & 0 & 219 & 116 & 316 & 175 & 362 & 116 & 268 & 167 & 354 \\
Th & 10682 & 10395 & 10666 & 10395 & 10626 & 10361 & 10666 & 10463 & 10634 & 10369 \\
Pu & 3712 & 3401 & 3535 & 3251 & 3470 & 3198 & 3535 & 3320 & 3482 & 3208 \\
MA & 441 & 423 & 427 & 409 & 423 & 405 & 427 & 414 & 423 & 405 \\
TRU & 4153 & 3824 & 3962 & 3660 & 3894 & 3603 & 3962 & 3735 & 3905 & 3614 \\
TRU consumption & 41.8 & \multicolumn{2}{c}{38.4} & 36.8 & 28.9 & 36.9 \\
CR & 0.9648 & 0.9669 & 0.9616 & 0.9681 & 0.9641 \\
\hline
\end{tabular}


about $250 \mathrm{~kg}$ of fertile Th232 is transmuted during each cycle, and it is transformed at least partially to fissile U233. In the considered design there is relatively no traces of U 235 and $U 238$ compared to $U 233$, and the production of higher actinides is reduced in comparison to cores based on uranium-plutonium cycle. We can find that mass of fissile materials stays almost constant and it agrees with observed conversion ratio. The net balance of plutonium indicates consumption, and it is similar for minor actinides (elements with atomic number >92), as they are effectively consumed. As a consequence, the burner side of the core comes into play with the burning TRU (Pu \& M A) isotopes mentioned. TRU consumption rate measures the amount of transuranic elements consumed per terawatt hour of power produced, during each cycle it was $\sim 28-42 \mathrm{~kg} / \mathrm{TWh}$ (Table 1 ) and it higher than for the reference design [9] which was 14 $\mathrm{kg} / \mathrm{TWh}$.

The calculated burnup increase at the end of each cycle was only $\sim 22 \mathrm{GW}$ d/tH M. Hence, after two cycles, fuel is far from reaching high burnups ( 150 GW d/tH M ) possible for fast reactors. Otherwise, the reference core design is not able to reach higher burnups as it will lose reactivity - see Figure 3. As an expected outcome of $\mathrm{U} 233$ production, there is no substantial reduction in $\mathrm{k}$ eff but also the thorium core can hypothetically operate without refuelling for more extended time period than reference design. Longer operation was tested for the first cycle, and reactivity margin is high enough that the core can still operate for at least 500days and possibly even more - see Figure 4.

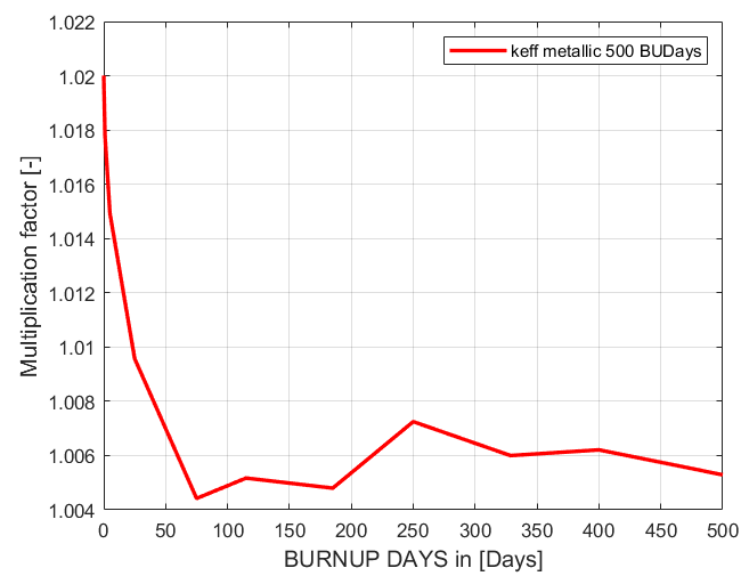

Fig. 4. First Fuel Cycle Simulation Effective M ultiplication Factor for 500 EFPDs.

\section{CONCLUSIONS AND SUMMARY}

A medium-sized 1000M W th Sodium Cooled Fast Reactor operating on Thorium M ixed Oxide (addition of Plutonium with Minor Actinides) fuel was studied. The emphasis was put into perspective the fuel cycles, actinides incineration and core inventory for the proposed core design.

The uniqueness of the studied thorium core is the fact that despite of high TRU consumption, we are not losing fissile material, and reactivity removal is substantially slower than in reference ABR uranium-plutonium core.
The irradiated thorium is transmuted to $\mathrm{U} 233$, which is not a TRU element but is fissile, and it induces additional positive reactivity. In traditional uraniumplutonium cycle to produce reactivity it would be necessary to produce plutonium and other TRU from uranium. What is more, irradiation of U-233 produces less higher actinides than U-235 and U-238 in fast spectra as it demands more neutron captures.

A point worth mentioning is: the TRU consumption is a goal for burner reactors as TRU elements are responsible for long term radiotoxicity of nuclear waste.

The presented design can hypothetically be used to burn the existing TRU stockpiles (plutonium and other higher actinides) and breed uranium from thorium. The criticality calculations showed that the reactor is critical throughout all five cycles with fissile material enrichment less than $20 \%$. The core can be operated well beyond its planned outage after 327 days, and it is expected that it could operate for at least 500 days with proper excess reactivity margin.

The study of the core safety parameters is ongoing, and it was not reported in this paper. In principle, it seems that the presence of thorium slightly degrades safety parameters. The presented results are preliminary and simulations were performed for relatively low neutron populations. In the next stage of the work simulations will be performed for much larger neutron populations to obtain results with substantially reduced M onte Carlo statistical errors. Further research will aim to assess safety aspects, study alternative core configurations, extended core operation and develop more optimized fuel cycles. The complete study of the whole fuel cycle with reprocessing would be a challenging endeavour.

\section{References}

1. OECD/nea, "Technology Roadmap Update for Generation IV Nuclear Energy Systems: Preparing Today for Tomorrow's Energy Needs" (2014).

2. Alan e. waltar, D. R. Todd, and P. V. Tsvetkov, Fast Spectrum Reactors, Springer (2013).

3. IAEA, "Liquid Metal Cooled Reactors: Experience in Design and Operation," Atomic Energy,(IAEATECDOC-1569) (2007); https://doi.org/IAEATECDOC-1569.

4. World nuclear news, "US DoE's Versatile Test Reactor Project"; http://www.world-nuclearnews.org/Articles/US-launches-test-reactor-project.

5. World nuclear association, "Thorium;" https://www.world-nuclear.org/informationlibrary/current-and-future-generation/thorium.aspx.

6. H. György and S. Czifrus, "The utilization of thorium in Generation IV reactors," Progress in Nuclear Energy, 93, pp. $306 \quad$ (2016); https://doi.org/10.1016/j.pnucene.2016.09.007.

7. H. György and S. Czifrus, "Investigation on the potential use of thorium as fuel for the Sodiumcooled Fast Reactor," Annals of Nuclear Energy, 103, pp. 238 (2017); https://doi.org/10.1016/j.anucene.2017.01.030.

8. N. E. Stauff et al., "Benchmark for Neutronic 
Analysis of Sodium-cooled Fast Reactor Cores with Various Fuel Types and Core Sizes," pp. 87 (2016).

9. T. K. Kim et al., "Annals of Nuclear Energy Core design studies for a $1000 \mathrm{MW}$ th Advanced Burner Reactor," Annals of Nuclear Energy, 36 (3), pp. 331, Elsevier Ltd (2009); https://doi.org/10.1016/j.anucene.2008.12.021.

10. R. Grandy, c., seidensticker, "Advanced Burner Reactor 1000MWth Reference Concept" (2007).

11. K. Insulander björk, Thorium Fuels for Light Water Reactors Steps towards commercialization (2015).

12. J. Leppänen, "PSG2 / Serpent - a Continuousenergy Monte Carlo Reactor Physics Burnup Calculation Code," pp. 157 (2011).

13. J. Leppänen, Development of a new Monte Carlo reactor physics code, in VTT Publications(640) (2007).

14. J. Leppänen et al., "The Serpent Monte Carlo code: Status, development and applications in 2013," Annals of Nuclear Energy, 82, pp. 142 (2015); https://doi.org/10.1016/j.anucene.2014.08.024.

15. C. Paper et al., "Evaluation of Large 3600MWth Sodium-Cooled Fast Reactor Neutronic OECD Benchmarks" (2014).

16. P. Darnowski and A. Mikołajczak, "Monte Carlo simulations of the 1000 MWth SFR OECD/NEA benchmark with SERPENT code," AIP Conference Proceedings, 2116 (July), pp. 450067 (2019); https://doi.org/10.1063/1.5114534.

17. P. Darnowski, "X-Core Nuclear Engineering Matlab Toolbox," GitLab Repostory; https://gitlab.com/darczu/x-core.

18. P. Stanisz, J. Cetnar, and M. Oettingen, "Radionuclide neutron source trajectories in the closed nuclear fuel cycle," Nukleonika, 64 (1), pp. 3 (2019); https://doi.org/10.2478/nuka-2019-0001. 